\title{
Traffic Routing in Urban Environments: the Impact of Partial Information
}

\author{
Lara Codecà, Raphaël Frank, Thomas Engel \\ Interdisciplinary Centre for Security, Reliability and Trust \\ University of Luxembourg, 2721, Luxembourg \\ lara.codeca@uni.lu,raphael.frank@uni.lu, thomas.engel@uni.lu
}

\begin{abstract}
There are many studies concerning the problem of traffic congestion in cities. One of the best accepted solutions to relieving congestion involves optimization of resources already available, by means of balancing traffic flows to minimize travel delays. To achieve this optimization, it is necessary to collect and process Floating Car Data (FCD) from vehicles. In this paper we evaluate the repercussions of partial information on the overall traffic view, and consequently on the outcome of the optimization. Our study focuses on the role of the user participation rate and the availability of Road Side Units to collect the FCD. By means of simulation we quantify the impact of partially-available information on the computation of route optimization, and how it impedes traffic flows. Our results show that even minor uncertainties can significantly impact routing strategies and lead to deterioration in the overall traffic situation.
\end{abstract}

\section{INTRODUCTION}

Vehicular traffic demand in metropolitan areas is increasing and drivers have to deal with traffic congestion and delays on a daily basis. Many studies have been conducted to find solutions to this issue and almost all focus on increasing the capacity of the transportation network. This can be accomplished by modifying the topology of the road network (e.g. adding new lanes), but this solution is not always practical and usually costly. For these reasons, the most frequently applied solution involves the optimization of the resources already available.

Navigation systems provide one step in the optimization process and have changed radically over the last decade. Initially they provided the fastest path between an origin and a destination, based solely on the route topology. Over time, more information regarding the traffic situation became available, not yet a live feed, but detailed enough to provide a solution that takes into account this information (e.g. Radio Data System - Traffic Message Channel (RDS-TMC) [1]) to provide the best route. Recently, the quality and quantity of information have allowed the possibility of dynamic routing. In this environment, it is possible to use different kinds of algorithms to optimize a variety of parameters (e.g. delay, path length, and fuel consumption). To apply dynamic routing, a bi-directional link between the vehicles and the source of the optimization is needed. This link is provided using the wireless technologies already available, which allow for collecting data and providing a service to the users.

This project is supported by the National Research Fund, Luxembourg.
In this paper we investigate the application of a mechanism able to optimize the usage of resources through a collaborative traffic management system that coordinates the routes of the vehicles involved. Route optimization is achieved by following the $1^{\text {st }}$ Wardrop principle of equilibrium. This mechanism uses real-time Floating Car Data (FCD) collected through Road Side Units (RSUs) that are part of a monitoring infrastructure. The FCD is aggregated in order to obtain an accurate and reliable overview of the traffic situation.

Several papers have proposed methods for sensing trafficrelevant information in form of FCD, such as [2]-[5]. Once the FCD has been collected, it must be aggregated to provide a reliable overview of the traffic situation of the monitored area. In the literature, many algorithms have been proposed to solve this issue [6]-[8]. In all the studies mentioned, there is an assumption that FCD provides a complete picture of the traffic situation. The number of On-Board Units (OBU) (e.g. navigation systems, micro-computers, and smartphones) has increased over the last years and the amount of FCD that can be retrieved on a large scale has grown. The use of this source of information has enabled the research community to explore other, more dynamic, approaches to improve traffic conditions. Nevertheless, to deploy systems that allow this kind of interactivity, it is necessary to investigate how the connectivity affects the behavior of the traffic monitoring system itself. We chose to use Wi-Fi because many cities have this type of network coverage provided in different ways [9], [10]. Moreover, cellular networks are increasingly overloaded with traffic due to the rapid growth of mobile broadband traffic. One of the solutions proposed for this problem is cellular traffic offloading through Wi-Fi networks [11], [12]. The completeness of the information is directly correlated with the level of user participation, the coverage in terms of infrastructure and connectivity of the monitored area, and the nature of the environment involved.

In this study we thoroughly evaluate, by means of simulation, how these parameters affect routing mechanisms and traffic congestion in metropolitan environments. The complexity of both the topology and the mobility models has an impact on the various aggregation methods and the level of detail and correctness of the represented traffic situation. The metropolitan environment is characterized by both short road segments and long arterial streets, interconnected by different 
types of intersections (e.g. traffic lights and right-before-left priority). Additionally the traffic comprises different types of vehicles, pedestrians and unexpected obstacles. To describe this complex environment many models have been developed in different fields of science [13]-[15].

Our result shows the behavior of the traffic monitoring system with different percentages of connectivity coverage and different numbers of active vehicles (i.e. the users that actively participate in the monitoring and routing system) in a Manhattan-grid topology. With less than $70 \%$ coverage, the routing mechanism fails, increasing the congestion instead of mitigating it. This behavior is because, without proper connectivity, the overview of the traffic situation is incomplete and the user equilibrium is computed with misleading information. We also show how the ratio between active and inactive RSUs (i.e. the connectivity coverage of the intersections) has a greater impact on the behavior than the actual user participation rate.

The rest of the paper is organized as follows. Section III defines the problem we study in this paper. Section IV presents in detail the elements in the infrastructure and the monitoring system. Section V describes the simulation environment and presents our results. Section II gives an overview of the state of the art, and in Section VI we draw a conclusion and provide directions for future work.

\section{RELATED WORK}

Issues such as FCD sampling and aggregation, RSU coverage, and vehicular traffic optimization have been widely studied in recent decades.

In [16] the authors present a framework enabling the use of big data analysis and cloud computing to process massive amounts of information in near real time. The only error taken into account is that related to the GPS position, while assuming information in terms of coverage of the territory.

Another example of FCD aggregation is presented in [17]. Here the authors integrate information collected from different sensors such as on-board camera, GPS and on-board diagnostics interface unit. They take into consideration the errors resulting from the image processing (qualitative errors) but not the possibility of having uneven coverage of the monitored area (quantitative errors).

In [18] the authors address the problem of RSU coverage and present a forwarding algorithm that relies only on local information. They use Vehicle-to-Vehicle (V2V) communication with $802.11 \mathrm{p}$ connectivity. Their result explores the RSU coverage extension and the improvement in terms of data dissemination. However they do not take into account the level of user participation, assuming that all the vehicles are equipped with an $\mathrm{OBU}$.

Another solution to the RSU coverage problem is proposed in [7]. The authors present a specific aggregation scheme in order to minimize the overall bandwidth necessary to transmit FCD. They assume a user participation rate of 5\% and use a genetic algorithm to identify the best position for the deployment of RSUs and achieve a strategic coverage of the

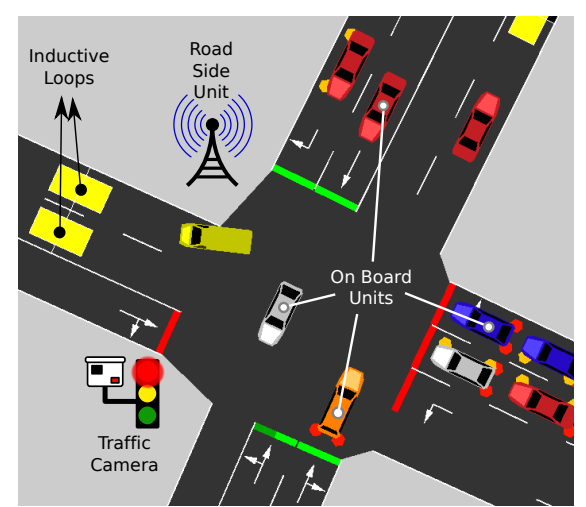

Fig. 1. Infrastructure example: Vehicles (with and without OBU), RSUs, Inductive Loops and Traffic Cameras.

territory that maximizes the effectiveness of the information gathered.

SOTIS [19], based on V2V communications, allows gathering, analyzing and broadcasting traffic information without the use of a central entity. With a user participation rate of $2 \%$, it manages to obtain roughly 30 -minute-old information concerning the area within around a $50-\mathrm{km}$ radius of the car. This kind of information is very useful in tracking major congestion in extra-urban environments, but cannot monitor the real-time traffic situation in urban areas. Here, the problem of sparse information is solved by keeping all data, regardless of its age.

In relation to the traffic optimization problem, [20] studies different kinds of vehicular route optimizations that utilize traffic information gathered from vehicles to estimate travel times and to find optimal routes. The authors study the difference between proactive and flow-based methods. They do not take the user participation rate into account and they assume that perfect information on the real-time traffic situation is available at decision time.

To the best of our knowledge, this paper is the first to investigate the impact of only having partial information available in terms of user participation rate and RSU coverage to deal with the problems of route optimization and traffic congestion.

\section{Problem Statement}

In every optimization problem the correctness and reliability of the information plays an important role. When the solution to the optimization problem must be provided at runtime, it is important to test the impact of partial information to discover boundaries and understand where particular behaviors originate. The reason we focused on this evaluation is to provide ground information for the deployment of the traffic management system. We take into consideration different types of information concerning traffic demand and the speed of specific vehicles. Figure 1 shows an example of these different sources of information. Floating Car Data (FCD) is collected through the On-Board Units (OBUs) in the vehicles and gathered by the Road Side Units (RSUs). In addition, the traffic demand and the global overview 
of the system are obtained through sources such as traffic cameras, inductive loop detectors, and historical information. In the literature there are many aggregation algorithms that allow different sources of traffic information to be used to obtain a global picture of the situation. Nonetheless, even if the infrastructure is already deployed, legal issues and other constraints make the information provided by inductive loops and traffic cameras hard to obtain, rarely live, and not always available. We decided to compare the behavior of the traffic monitoring system in two different settings. The first considers only FCD, while the second considers the overview computed with all the aggregated sources, FCD included.

\section{TRAFFIC MONITORING INFRASTRUCTURE}

\section{A. Wardrop Equilibrium}

The optimization of the traffic flows is based on Wardrop equilibrium [21], which provides an explanation accepted [22] as common behavior to describe how the trips spread over different routes due to congested traffic conditions. The routing mechanism implemented here is based on the selfishuser-centered policy defined by Wardrop's first principle of equilibrium. This principle states that every player (selfishly) selects a route that minimizes the travel cost between source and destination. Wardrop's second principle posits that users minimize the total travel time in the system. We use Dijkstra's shortest-path algorithm [23] with a dynamic edge cost to compute the route with the minimum delay. The cost of the edge is the Estimated Travel Time (ETT) of the relevant road segment and this value changes dynamically over time. To compute $E T T_{s}$ for a road segment $s$ we use its length, $l_{s}$, divided by the average speed $\overline{v_{s}}$ of all vehicles currently traveling on that segment:

$$
\overline{v_{s}}=\frac{1}{n_{s}} \sum_{i=1}^{n_{s}} v_{i}, E T T_{s}=\frac{l_{s}}{\overline{v_{s}}} .
$$

where $n_{s}$ is the number of vehicles on segment $s$ and $v_{i}$ is the speed of vehicle $i$. A route is composed of multiple segments. Its total cost $E T T_{r}$ is calculated as the sum of all the segments composing the route. The minimum-delay route between the source $s$ and the destination $d$ is the one that minimizes ETT. Upon receiving a request from the vehicle, the system provides this as the best route.

We have shown in a previous paper [24] the reasons why we support the use of a selfish, user-centered approach against a global one. The main reason is related to the the constraints imposed by the global one. The optimum in terms of minimum overall delay can be achieved only with the global and fully coordinated approach, but this would imply that all the vehicles in the monitored area are part of the system and that they are $100 \%$ compliant with the instructions provided.

\section{B. Data gathering infrastructure}

An OBU is any device (e.g. smartphone, single-board computer, embedded system, etc.) deployed in a vehicle, able to collect data from sensors, and having connectivity capabilities. The FCD sampled by the OBU consist of GPS position, speed and direction. In our topology, this data is gathered by the RSUs located at the intersections. Even assuming that all FCD is reliable and correct, the consistency of the overall traffic view (computed with the FCD) with the real traffic situation depends on the numbers of active vehicles that cooperate with the traffic monitoring system, and on the proportion of intersections covered by the RSUs.

To study the impact of the RSUs' coverage on the real-time traffic overview we use a 10x10 Manhattan grid topology with edges of $4.5 \mathrm{~km}$. All streets are bidirectional and have the same priority rules. Each intersection follows the rightbefore-left priority rule, and is equipped with a $\mathrm{Wi}-\mathrm{Fi}$ access points acting as RSUs. Our aim is to provide a ground study with general results. By using a regular topology, we are able to minimize the impact of RSU coverage patterns, which can vary depending on intersection location. All communications are based on a Vehicle-to-Infrastructure (V2I) paradigm in which the vehicles in the monitored area are able to communicate with a local RSU using their OBU. The traffic management protocol is that presented in [24] and is divided into two parts: Information Beaconing and Route Management. Both of these parts require a reliable representation of the traffic situation.

Remark: The following two sections explain the realtime data aggregation IV-C and the traffic management protocol IV-D. These two aspects are not dependent on each other. The real-time data aggregation concerns the dataset and its metrics. The traffic management protocol concerns the route optimization mechanism.

\section{Real-Time Data Aggregation}

We define the Real-Time Traffic Situation (RTTS) as the reliable overview of the system provided through the aggregation of all the different data pertaining to vehicular traffic. We use this information to compute the optimum user equilibrium and decide the best route for a vehicle. The two scenarios that we explore in this paper are differentiated by the kinds of information used to build the RTTS. Figure 2 depicts the kind of information that is used to compute the RTTS. More precisely, we define: $O F C D$ (Only FCD) (green arrow labeled 1), the dataset composed of information collected in real-time through the OBUs of the vehicles; ASOD (All Sources Of Data) (red arrow labeled 2 ), the dataset composed of all the aggregated information gathered from RSUs, inductive loops, traffic cameras, both stationary (infrastructure) and floating (vehicle) data. Specific to this implementation, in the scenario in which the RTTS is computed with the OFCD dataset, the aggregation consists of the average of the samples specific to a segment. Every segment has a predefined limited-time buffer and all samples prior to that time window are discarded. In case of lack of information, the values used to compute the deterministic free flow are those provided by the topology (maximum speed allowed and length of the segment). Regarding the scenario with the RTTS built from the ASOD dataset, we do not need to implement any specific aggregation method since the 


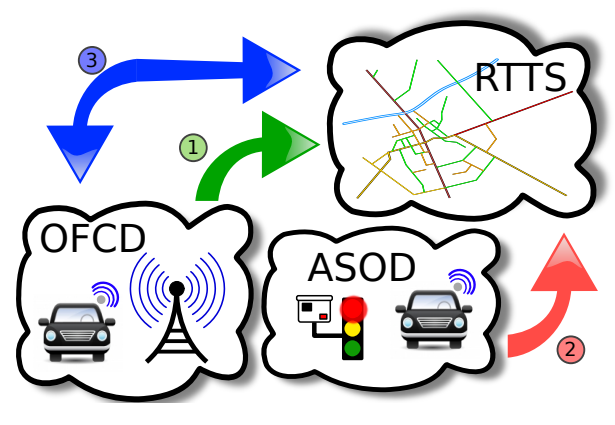

Fig. 2. Traffic monitoring system and routing mechanism.

simulator that we use has exact knowledge of the number of vehicles in every segment and their velocities.

\section{Traffic Management Protocol}

The traffic management protocol represented in Figure 2 can be summarized as follows. Information Beaconing (green arrow labeled 1) the part of the protocol designed to sample and collect traffic metrics (location, direction and speed) from the OBU. Every 60 seconds, the OBU sends the metrics to the closest RSU located at an intersection in communication range. The RSUs aggregate these metrics dynamically to update the RTTS. The Route Management part of the protocol is designed to redistribute the vehicular traffic to optimize the route. The protocol interaction (blue arrow labeled 3) for every user is the following: (i) the OBU sends the route request containing the current location and desired destination to the local RSU, (ii) the optimal route is computed using the Wardrop equilibrium (see IV-A) applied to the RTTS and sent as a reply to the OBU, (iii) the OBU receives a route update every 60 seconds.

\section{A. Simulation environment}

\section{Evaluation}

We built the topology described in IV-B in a simulated environment using OMNet++ [25] for the wireless network and SUMO [26] for the vehicular mobility model. The bidirectional coupling mechanism between the network and mobility model is provided by VEINS [27]. Each intersection is equipped with a Wi-Fi access point acting as a RSU. Concerning vehicular mobility, SUMO provides different models that are able to simulate specific patterns for different situations. We chose the Krauss car-following model to simulate an urban traffic flow; the parameters we use for the vehicles are those proposed in the original document [28]. Each vehicle has a source and a destination randomly chosen among all the edges of the Manhattan topology. To compute the route from source to destination, we use duarouter [29], a tool provided by SUMO that uses Dijkstra's algorithm to identify the path having minimal cost. In this case, cost is determined using the length, maximum speed, and priority of the segment.

\section{B. Evaluation parameters}

We ran a scenario with 10,000 vehicles, all starting their trip at the beginning of the simulation. The aim is to measure the time necessary for all of them to reach their destinations.

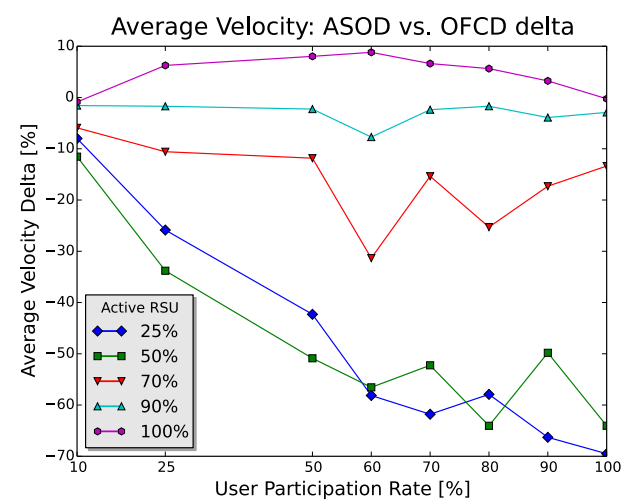

Fig. 3. Average speed comparison between OFCD and ASOD scenarios varying User participation rate and RSUs coverage.

We collected statistics on vehicular mobility (e.g. average speed, waiting time of each vehicle, and density) and RSU coverage. As explained in Section III, the aim of this work is to evaluate the impact of partial information on the solution proposed by the routing protocol. The completeness of the overview given by the information depends on the different participation rates of the vehicles and RSU coverage at the intersections. These are very important parameters to consider when it comes to the deployment of the system. For our evaluation we used: $10 \%, 25 \%, 50 \%, 60 \%, 70 \%$, $80 \%, 90 \%$, and $100 \%$ rates both for RSU coverage and user participation. The intersections covered by the RSUs and the vehicles equipped with the OBUs were randomly selected. To show how the use of partial information influences the routing mechanism, we compared the behavior of the protocol with the two different sets of information collected and aggregated into the RTTS. As explained in Section IV-C, in one scenario, the RTTS is computed using the OFCD dataset and in the other, the ASOD dataset. As for the interval between two routing requests (and consequently the beaconing interval and the route update) sent from a given vehicle, we fixed it at 60 seconds. Both vehicular mobility and overall traffic change slowly. It has been proven that using a smaller time interval does not significantly alter the routing mechanism's performance. The evaluation of this parameter and a detailed explanation can be found in [24].

\section{Results}

Speed: Figure 3 gives a quantitative comparison between the average speed of all the vehicles in the two different scenarios. In the first, the RTTS is computed using the ASOD dataset. The measured average speed is used as a basis for the comparison. This represents the ideal situation in which we have a global overview of the system even without the full cooperation from the users (i.e. the traffic information is complete and reliable even without active vehicles, due to traffic cameras, etc.). In the second scenario the RTTS is computed using only the FCD collected from the active RSUs. The value represented in the graph is the delta (as a percentage) between the average speed measured in the second scenario compared with the first. We chose to plot 


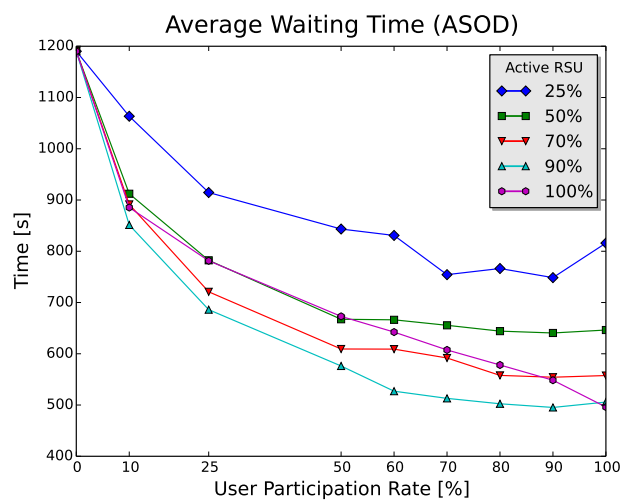

(a) Average waiting time, ASOD dataset

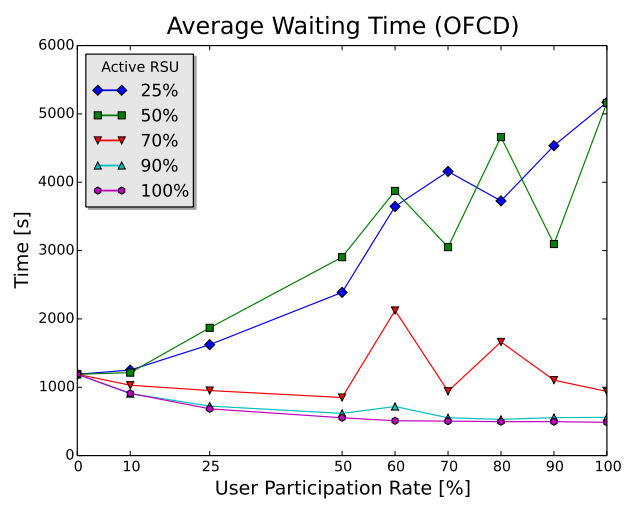

(b) Average waiting time, OFCD dataset

Fig. 4. Average waiting time.

the more representative percentages from all the experiments. We hypothesized that a routing system that takes decisions based on a dataset with partial information will behave differently based on the completeness of the dataset itself. We investigated the boundary between the normal behavior expected from the routing mechanism and the compromised behavior caused by the use of partial information. We found the threshold to be around $70 \%$ of intersection covered by RSUs. It can be seen that, without ASOD information, the coverage plays an important role. It is possible to see that with $80 \%$ or more coverage, the performance of the routing mechanism is consistent in both scenarios, even with low user participation. When the coverage is $70 \%$ or less, the information collected by the RSUs can be misleading, and the protocol fails to relieve congestion. This behavior is due to the fact that when the ratio of intersections covered by the RSUs is insufficient, the overview of the situation is incomplete and the user equilibrium is computed with misleading information. In this graph it is easy to see that the user participation rate has only a minor impact on the behavior of the routing mechanism compared with the percentage of intersections with active RSUs.

Waiting Time: Another aspect that we analyzed is the impact of partial information on the average waiting time encountered by the vehicles. We ran a scenario in which the vehicles provide no information and the routing mechanism

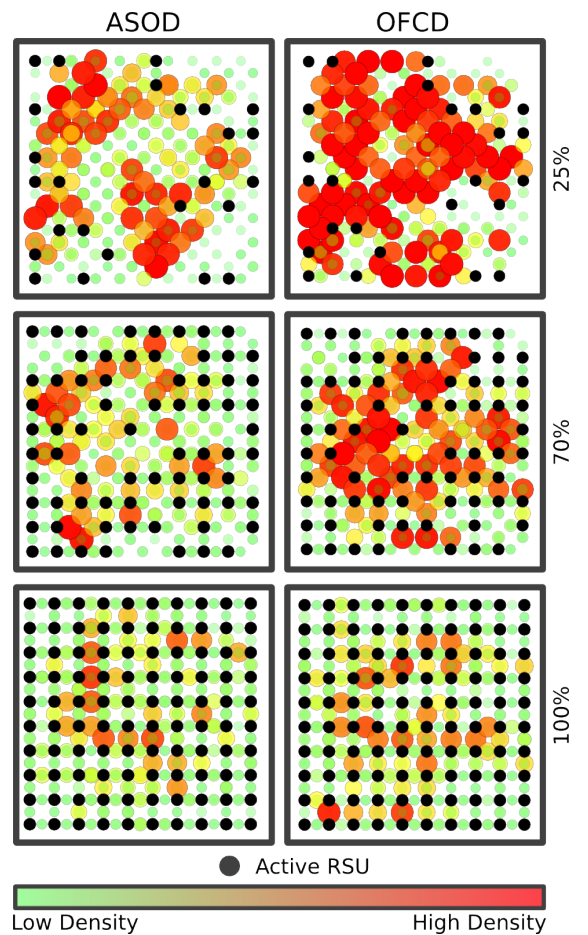

Fig. 5. Vehicular Density and RSU Coverage in different scenario at the same simulation time.

is not in place to provide a background truth for comparison purposes. We define waiting time as the amount of time during which the vehicle is stopped. More precisely, Figure 4(a) shows the average waiting time encountered by each vehicle in the scenario with complete information. It can be seen that, independent of the coverage, even with $25 \%$ participation, there is an improvement due to the use of complete information. On the other hand, in Figure 4(b) the two different behaviors become visible. With less than $70 \%$ coverage, the trends again show erratic behavior among the vehicles.

Density: In Figure 5 we show the vehicular density on the grid, comparing the same routing mechanism applied to two different RTTSs, one computed with ASOD, the other with OFCD. The black dots represent the active RSUs. On the grid, the size and color of the dots represents the occupancy of a specific segment. All these snapshots of the traffic situation are taken at the same time (second $2250)$ with the same user participation rate $(100 \%)$. With $100 \%$ RSU coverage, the level of density is comparable in both scenarios. The threshold of $70 \%$ discovered previously reveals a visible difference between ASOD and OFCD in vehicular density. The segments leading to intersections not covered by an active RSU, in case of FCD only, are more congested than the others. This behavior is similar but less visible with ASOD. This is the case because the lack of coverage at an intersection not only decreases the amount of FCD, but also prevents route updates from reaching interested vehicles. This behavior becomes more accentuated when only $25 \%$ of intersections are covered by active RSUs. While with ASOD, the situation is still acceptable, for OFCD, the 
situation is worsened by the fact that the few route updates that are received, may be sub-optimal due to the use of partial information.

Remarks: The problems highlighted by the use of partial information can be further investigated in a number of ways. Firstly, employing advanced heuristics to compute the RTTS is a promising approach to avoiding errors when dealing with incomplete information. Secondly, it could be fruitful to investigate the use of probabilistic algorithms to compute user equilibrium and route updates. Nevertheless, our works indicates that, with a deterministic aggregation that assumes free flow in case of missing information and a deterministic routing mechanism, the completeness of the information plays a major role and must be taken into account during the deployment of such a system. In addition, partial information can be a threat to security and may compromise the effectiveness of the traffic management system.

\section{CONCLUSION AND FUTURE WORK}

In this paper we evaluated the behavior of a traffic monitoring system that has to deal with partial information. The mechanism is able to update the route of the vehicles, and so relieve traffic congestion, but requires a reliable realtime traffic overview of the system to take decisions. The correctness of this overview depends on various parameters such as the user participation rate and the percentage of intersections covered by RSUs.

Our results shows that with less than $70 \%$ of RSU coverage, the traffic overview does not coherently represent the real traffic situation and it can represent security issues (e.g. if the information is altered by malicious attacks or physical damages in the infrastructure). This behavior does not vary significantly depending on the user participation rate. This result indicates that the percentage of intersections covered by RSUs plays a major role in information-gathering and route updates. With insufficient coverage, the routing mechanism fails to relieve congestion and, due to misleading information, may worsen it.

In the future we plan to further investigate how to mitigate the impact of partial information due to malicious attacks. The current paper uses a deterministic aggregation method that, in case of missing information, assumes free flow. Further studies can be done with probabilistic aggregation methods that, for example, can take into account the traffic situation in the surrounding area in order to estimate the missing traffic information.

\section{REFERENCES}

[1] D. Kopitz and B. Marks, RDS: the radio data system. Artech House, 1999.

[2] R. Frank, "Energy-Efficient Rate-Adaptive Passive Traffic Sensing using Smartphones," Micro-NanoMechatronics Hum. Sci. (MHS), 2012 Int. Symp. on., 2013.

[3] J. Paek, J. Kim, and R. Govindan, "Energy-efficient rate-adaptive GPSbased positioning for smartphones," Proc. 8th Int. Conf. Mob. Syst. Appl. Serv., vol. 223-224, pp. 299-314, 2010.

[4] A. Kovacheva, R. Frank, and T. Engel, "LuxTraffic: A collaborative traffic sensing system," Local Metrop. Area Networks (LANMAN), 2013 19th IEEE Work., pp. 1-6, Apr. 2013.
[5] X. Li, W. Shu, M. Li, and H. Huang, "Performance evaluation of vehicle-based mobile sensor networks for traffic monitoring," Veh. Technol. IEEE Trans., 2009.

[6] C. Lochert, B. Scheuermann, and M. Mauve, "Probabilistic Aggregation for Data Dissemination in VANETs," Proc. fourth ACM Int. Work. Veh. ad hoc networks., 2007.

[7] C. Lochert and B. Scheuermann, "Data Aggregation and Roadside Unit Placement for a VANET Traffic Information System," Proc. fifth ACM Int. Work. Veh. Inter-NETworking., 2008.

[8] B. Scheuermann and C. Lochert, "A Fundamental Scalability Criterion for Data Aggregation in VANETs," Proceedings 15th Annu. Int. Conf. Mob. Comput. networking., 2009.

[9] C. Middleton and A. Potter, "Is it good to share? A case study of the FON and Meraki approaches to broadband provision," Proc. ITS 17th Bienn. Conf., pp. 163-175, 2008.

[10] C. Middleton and A. Bryne, "An exploration of user-generated wireless broadband infrastructures in digital cities," Telemat. Informatics, vol. 25, pp. 1-31, 2011.

[11] B. Han and A. Srinivasan, "Mobile Data Offloading in Metropolitan Area Networks," ACM SIGMOBILE Mob. Comput. Commun., 2010.

[12] S. Dimatteo, P. Hui, B. Han, and V. O. Li, "Cellular Traffic Offloading through WiFi Networks," 2011 IEEE Eighth Int. Conf. Mob. Ad-Hoc Sens. Syst., pp. 192-201, Oct. 2011.

[13] G. Wong and S. Wong, "A multi-class traffic flow model an extension of LWR model with heterogeneous drivers," Transp. Res. Part A Policy Pract., vol. 36, no. 9, pp. 827-841, Nov. 2002.

[14] G. Newell, "A simplified car-following theory: a lower order model," Transp. Res. Part B Methodol., vol. 36, pp. 195-205, 2002.

[15] C. F. Daganzo and N. Geroliminis, "An analytical approximation for the macroscopic fundamental diagram of urban traffic," Transp. Res. Part B Methodol., vol. 42, no. 9, pp. 771-781, Nov. 2008.

[16] Q. Li, T. Zhang, and Y. Yu, "Using cloud computing to process intensive floating car data for urban traffic surveillance," Int. J. Geogr. Inf. Sci., vol. 25, no. 8, pp. 1303-1322, 2011.

[17] S. Messelodi, C. M. Modena, M. Zanin, F. G. De Natale, F. Granelli, E. Betterle, and A. Guarise, "Intelligent extended floating car data collection," Expert Syst. Appl., vol. 36, no. 3, pp. 4213-4227, Apr. 2009.

[18] P. Salvo, F. Cuomo, A. Baiocchi, and A. Bragagnini, "Road Side Unit coverage extension for data dissemination in VANETs," 2012 9th Annu. Conf. Wirel. On-Demand Netw. Syst. Serv., pp. 47-50, Jan. 2012.

[19] L. Wischoff, A. Ebner, and H. Rohling, "SOTIS-a self-organizing traffic information system," Veh. Technol. Conf. 2003., vol. 4, pp. 24422446, 2003.

[20] F. Angius, M. Reineri, C. Chiasserini, M. Gerla, and G. Pau, "Towards a realistic optimization of urban traffic flows," 2012 15th Int. IEEE Conf. Intell. Transp. Syst., pp. 1661-1668, Sep. 2012.

[21] J. Wardrop, "Road Paper. Some Theoretical Aspects of Road Traffic Research," ICE Proc. Eng. Div., 1952.

[22] J. Correa and N. Stier-Moses, "Wardrop equilibria," Wiley Encycl. Oper. Res. Manag. Sci., pp. 1-13, 2011.

[23] J. Chen, "Dijkstra's shortest path algorithm," J. Formaliz. Math., 2003.

[24] L. Codeca, R. Frank, and T. Engel, "Improving Traffic in Urban Environments applying the Wardrop Equilibrium," Work. Veh. Commun. Appl., 2013.

[25] A. Köpke, M. Swigulski, K. Wessel, D. Willkomm, P. T. K. Haneveld, T. E. V. Parker, O. W. Visser, H. S. Lichte, and S. Valentin, "Simulating wireless and mobile networks in OMNeT++ the MiXiM vision," in Proc. 1st Int. Conf. Simul. tools Tech. Commun. networks Syst. Work., 2008, pp. 71:1-71:8.

[26] M. Behrisch, L. Bieker, J. Erdmann, and D. Krajzewicz, "SUMO Simulation of Urban MObility - an Overview," in SIMUL 2011, Third Int. Conf. Adv. Syst. Simul., 2011, pp. 55 - 60.

[27] C. Sommer, R. German, and F. Dressler, "Bidirectionally coupled network and road traffic simulation for improved IVC analysis," Mob. Comput. IEEE Trans., vol. 10, no. 1, pp. 3-15, 2011.

[28] S. Krauß, "Microscopic modeling of traffic flow: Investigation of collision free vehicle dynamics," Dtsch. Zent. fur Luft und Raumfahrt Forschungsbericht, 1998.

[29] F. Kaisser, C. Gransart, M. Kassab, and M. Berbineau, "A framework to simulate VANET scenarios with SUMO,” Opnetwork, 2011. 